\title{
Baryon Magnetic Moments and Axial Coupling Constants with Relativistic and Exchange Current Effects
}

\author{
K. Dannbom, C. Helminen and D.O. Riska \\ Department of Physics, University of Helsinki, 00014 Finland
}

\begin{abstract}
The large relativistic corrections to the constituent quark current operators improve the predictions for the axial couplings of the baryons, but worsen those for their magnetic moments. The exchange current corrections that are associated with flavor and spin dependent hyperfine interactions between the quarks with a form suggested by pseudoscalar meson exchange can compensate the relativistic corrections to the baryon magnetic moments. This is demonstrated by a calculation of the magnetic moments of the non-strange and strange baryons using wave functions and exchange current operators, which correspond to a recent phenomenological spin- and flavor dependent interquark interaction model, which yields a spectrum close to the empirical one.
\end{abstract}




\section{Introduction}

As the constituent quarks are much lighter than the nucleon, both their electromagnetic and the axial current operators have significant relativistic corrections, if the constituent quarks are treated as Dirac particles [1]. While the magnitude of these relativistic corrections depends on the average velocity of the confined quarks and thus on the model for the hyperfine interaction between the quarks [2], their effect is to reduce the magnitude of the predicted values of both the axial coupling constants and the magnetic moments of the baryons that are given by the static quark model. While this correction reduces the standard overprediction of the axial current coupling constants of the nucleons (5/3 vs 1.24) and the strange hyperons it worsens the mostly satisfactory predictions for the magnetic moments of the baryons that are obtained with the static quark model in the impulse approximation. We shall show here that the exchange current correction that is associated with spin- and flavor dependent interactions between the quarks, with the same operator structure as that of the pseudoscalar meson octet exchange interaction between the quarks can compensate for the relativistic correction in the latter case, while leaving it operative in the case of the axial coupling constants. This then suggests a way to obtain an at least qualitatively satisfactory simultaneous description of both the magnetic moments and the axial coupling constants although a quantitative description would require a completely relativistic quantum mechanical approach.

There are several different dynamical mechanisms that can give rise to flavor dependent interactions between constituent quarks. To these belong the interaction mediated by pseudoscalar octet exchange between the quarks [3, 4. The coupling of the light pseudoscalar meson octet, which forms the octet of Goldstone bosons of the spontaneously broken approximate chiral symmetry of QCD, to the constituent quarks is probably a significant factor in the explanation of the spectra of baryons and it also resolves several of the problems with the naive quark model and the measured spin- and strangeness content of the proton [5]. For the present issue the salient point is that the flavor dependent octet boson exchange interaction, by the requirement of current conservation, implies the presence of octet vector two-body exchange magnetic moment operators. As these increase the magnitudes of the magnetic moments of the baryons that are predicted in the static quark 
model [6], they in principle should counteract the reduction caused by the relativistic correction. There are no corresponding pure pseudoscalar octet exchange contributions to the axial current of the baryons.

We shall here construct the pseudoscalar boson exchange current so as to be consistent with and to satisfy the continuity equation with the pseudoscalar boson exchange interaction even when this is modified phenomenologically at short range. In the calculations we employ the explicit phenomenological model for the hyperfine interaction between the quarks given in ref. [4], which yields a satisfactory description of the nucleon and $\Delta$ spectra in combination with a static linear confining interaction. As the volume integral of that interaction model does not vanish, only part of it can be interpreted as being due to pseudoscalar exchange mechanisms. The remaining part is treated $\mathrm{p}$ henomenologically, using an appropriate exchange current operator for an interaction with nonvanishing volume integral.

The construction of the flavor octet exchange current operator is nonrelativistic. That relativistic corrections to the exchange current operator also are important is however evident from the fact that the octet vector exchange currents, which are the quark level analogues of the two-nucleon isovector exchange currents, are inoperative in the case of the baryon decuplet, the flavor states of which by definition are symmetric. In order to compensate for the large relativistic corrections to the magnetic moment of the decuplet baryons, as e.g. the $\Omega^{-}$, we therefore also consider the lowest order relativistic corrections to the exchange magnetic moment operators, which have flavor symmetric terms. These are the quark level analogues of the (small) isoscalar two-nucleon exchange current operators, and arise from excitation of virtual $q \bar{q}$-pairs. It proves possible to estimate the matrix elements of these relativistic exchange magnetic moment operators directly from the corresponding matrix element of the pseudoscalar exchange hyperfine interaction, which can be determined directly from the $N \Delta(1232)$ splitting provided that one in the case of the strange hyperons takes into account the different constituent quark masses.

In the present calculation we employ for the proton and the neutron the 3 quark wave function obtained in ref. [4] by solving the Faddeev equations with a spin- and flavor dependent model for the hyperfine interaction 
between the quarks and a linear confining potential, which yields a baryon spectrum that is close to the empirical one. For the non-strange and the strange hyperons we use a phenomenological wave function model [3], which is fitted to the numerically exact one for the nucleons, but which contains the masses of the constituent quarks as explicit parameters and therefore permits extrapolation to the case of baryons with strange quarks. As the quark Hamiltonian is purely nonrelativistic we have to treat the required relativistic corrections to the exchange current operators in an ad hoc way, which is suggested by those that appear in the single quark current operators. The present results therefore remain qualitative, but even so we find that the exchange current contributions that are implied by the quark-quark interaction model in ref. [4] are more than sufficient to compensate the reduction of the impulse approximation values for the magnetic moments, which are caused by the relativistic corrections.

This paper is divided into 6 sections. In section 2 we discuss the relativistic corrections to the baryon current couplings. The static pseudoscalar exchange current magnetic moment operator is derived in section 3. In section 4 we derive the lowest order relativistic correction to the pseudoscalar exchange current operators, which includes flavor symmetric components. In section 5 we consider the exchange current corrections that are associated with the part of the phenomenological interaction, which cannot be interpreted as being due to pseudoscalar exchange mechanisms. A summarizing discussion is given in section 6 .

\section{Relativistic constituent quark currents}

Under the assumption that the constituent quarks can be treated as point

Dirac particles without anomalous terms, their electromagnetic and axial current operators are

$$
\begin{gathered}
<p^{\prime}\left|J_{\mu}(0)\right| p>=i e \bar{u}\left(p^{\prime}\right) \gamma_{\mu}\left[\frac{1}{2} \lambda_{3}+\frac{1}{2 \sqrt{3}} \lambda_{8}\right] u(p), \\
<p^{\prime}\left|A_{\mu a}(0)\right| p>=i g_{A}^{q} \bar{u}\left(p^{\prime}\right) \gamma_{\mu} \gamma_{5} \frac{\lambda_{a}}{2} u(p) .
\end{gathered}
$$


Here $g_{A}^{q}$ is the axial current coupling of the constituent quarks, which in the large color limit is 1 [7], and with inclusion of the lowest $1 / N_{c}$ correction is $g_{A}^{q} \simeq 0.87$ [8]. An argument for the absence of anomalous terms in the electromagnetic current (2.1a) has been given in ref. [9].

In terms of Pauli spinors these operators reduce to

$$
\begin{gathered}
\vec{J}=e\left[\frac{1}{2} \lambda_{3}+\frac{1}{2 \sqrt{3}} \lambda_{8}\right] \frac{1}{\sqrt{1+\vec{v}^{2}}}\left\{\left[\vec{v}+i \frac{\vec{\sigma} \times \vec{q}}{2 m_{q}}\right]\right. \\
\left.-\frac{\sqrt{\left(\vec{P}+(\vec{q} / 2)^{2}\right.}-\sqrt{\left(\vec{P}-(\vec{q} / 2)^{2}\right.}}{2 m_{q}\left[1+\sqrt{1+\vec{v}^{2}}\right]}\left[\frac{\vec{q}}{2 m_{q}}+i \vec{\sigma} \times \vec{v}\right]\right\}, \\
\overrightarrow{A_{a}}=-g_{A}^{q} \frac{\lambda_{a}}{2} \vec{\sigma}\left\{\left(1-\frac{2}{3}\left(1-\frac{1}{\sqrt{1+\vec{v}^{2}}}\right\} .\right.\right.
\end{gathered}
$$

Here the velocity operator is defined as $\vec{v}=\vec{P} / m_{q}$, with $\vec{P}=(\vec{p}+\vec{p}) / 2$ and $m_{q}$ is the mass of the constituent quark. The momentum transfer is denoted $\vec{q}=\vec{p}-\vec{p}$. Note that in the expression for the electromagnetic current $(2.2 \mathrm{a})$ the limit $\vec{p}=\vec{p}^{\prime}$ has been taken in all terms, where it does not affect the derivation of the magnetic moment operator $\vec{\mu} \equiv-\frac{i}{2}(\nabla \times \vec{q})_{\vec{q}=0}$. The spin term in the magnetic moment operator that corresponds to the electromagnetic current operator $(2.2 \mathrm{a})$ is then

$$
\vec{\mu}_{\text {spin }}=\frac{e}{2 m}\left[\frac{1}{2} \lambda_{3}+\frac{1}{2 \sqrt{3}} \lambda_{8}\right] \frac{\vec{\sigma}}{\sqrt{1+\vec{v}^{2}}}\left\{1-\frac{1}{3}\left(1-\frac{1}{\sqrt{1+\vec{v}^{2}}}\right)\right\} .
$$

To lowest order in $\vec{v}^{2}$ the expressions for the spin magnetic moment and axial current operators are then

$$
\begin{gathered}
\vec{\mu}_{\text {spin }}=\frac{e}{2 m}\left[\frac{1}{2} \lambda_{3}+\frac{1}{2 \sqrt{3}} \lambda_{8}\right] \vec{\sigma}\left(1-\frac{2 \vec{v}^{2}}{3}\right), \\
\vec{A}_{a}=-g_{A}^{q} \frac{\lambda_{a}}{2} \vec{\sigma}\left(1-\frac{\vec{v}^{2}}{3}\right) .
\end{gathered}
$$

The relativistic $\vec{v}^{2}$ correction is large in the case of the constituent quarks, the masses of which are of the order 300-400 MeV. Its order of magnitude is easiest to estimate with the harmonic oscillator model for the quark confinement, in which case 


$$
<\vec{v}^{2}>=\frac{\omega}{m_{q}}=\frac{1}{m_{q}^{2} r^{2}},
$$

where $\omega$ is the oscillator parameter and $r$ the mean radius $\left(r=1 / \sqrt{m_{q} \omega}\right)$. As $r \sim 0.86 \mathrm{fm}$ for the baryons, the latter expression (2.5) gives $<\vec{v}^{2}>=0.45$ if $m_{q}=340 \mathrm{MeV}$, which is an appropriate value for the nonstrange constituent quarks. This shows that the relativistic correction to the magnetic moments amounts to a large reduction of the values predicted by the static quark model. The large value of $\left\langle\vec{v}^{2}\right\rangle$ also shows that the $v / c$ expansion is unreliable, and that the unapproximated expressions (2.2b) and (2.3) have to be employed. With $m_{u}=340 \mathrm{MeV}$ the empirical radius $0.86 \mathrm{fm}$ corresponds to $\omega=154 \mathrm{MeV}$ in the harmonic oscillator model. This is an appropriate value under the assumption that the hyperfine interaction is entirely due to pseudoscalar boson exchange and that it can be treated in first order perturbation theory in the oscillator model. When the 3-quark system is solved with a linear confining potential and with full account of the pseudoscalar exchange interaction to all orders the wave function is much more compact, with a matter $r m s$ radius of $0.47 \mathrm{fm}$ [ [⿴囗十]. In the oscillator model this corresponds to an oscillator frequency of $\sim 540 \mathrm{MeV}(2.5)$ (if the hyperfine interaction is neglected). We shall here employ the known exact wave function for this model for the non-strange baryons [四] and an oscillator model wave function with this oscillator frequency for the strange hyperons, as this should provide the most appropriate extrapolation to the states with the heavier strange constituent quarks for which we use the mass $460 \mathrm{MeV}$ [3].

Consider first the magnetic moments of the ground state baryons. In the impulse approximation the static quark model expressions for the magnetic moments are linear combinations of the ratios of the nucleon to the up, down and strange constituent quark masses (Table 1). With the usual approximation that the constituent masses of the up and down quarks are equal, the two ratios to be considered are $m_{N} / m_{u}$ and $m_{N} / m_{s}$. In Table 1 we have taken the relativistic correction in the expression (2.3) for the magnetic moment operator into account by the replacements 13.

$$
\frac{1}{m} \rightarrow \frac{1}{m^{*}}=\frac{1}{m}<\frac{1}{\sqrt{1+\vec{v}^{2}}}\left(1-\frac{1}{3}\left(1-\frac{1}{\sqrt{1+\vec{v}^{2}}}\right)\right)>
$$

in the quark masses in the impulse approximation expressions. 
The results in column I in Table I show that all the measured baryon magnetic moments, with exception of the $\Delta^{++}$are satisfactorily $( \pm 15 \%)$ predicted in the static impulse approximation in the absence of the relativistic correction. The calculation of the neutron and proton magnetic moments was done with the exact 3 quark wavefunction of ref. 四. The same relativistic reduction would in the oscillator model be obtained with a wave function with an "effective" oscillator frequency of $540 \mathrm{MeV}$. In the absence of a similar exact wave function for the hyperons we have therefore used the oscillator model with $\omega_{\text {eff }}=540 \mathrm{MeV}$ to estimate the relativistic corrections to the magnetic moments of the strange hyperons and the $\Delta$ :s. Including the relativistic correction reduces the predicted magnetic moment values by $20-30 \%$, and notably worsens the agreement with the experimental values. We shall show in the following section that the exchange current corrections can compensate these reductions in most cases.

In Table 2 the axial vector couplings of the octet baryons are listed. With the exception of $g_{A}\left(\Xi^{-} \rightarrow \Sigma^{0}\right)$ the empirical values are considerably smaller in magnitude than the values predicted by the static quark model. The static quark model predictions are most conveniently given as the $D, F$ coefficient values

$$
D=1, \quad F=\frac{2}{3} .
$$

The corresponding empirical values are 0.77 and 0.45 [14], which are $23 \%$ and $32 \%$ smaller than the static quark model values respectively. The static quark model values as obtained with $g_{A}^{q}=1$ are given in column II of Table 1. The corresponding values that are obtained with the relativistic correction in eq. (2.2b) taken into account are listed in column III. The exact wave function of ref. [⿴囗大 was used for the calculation of the relativistic correction to $g_{A}(n \rightarrow p)$. In the case of the strange hyperons we again employed the oscillator wave function model with $\omega=540 \mathrm{MeV}$, which leads to the same result as the exact wave function in the case of $g_{A}(n \rightarrow p)$. Here we have used the average of the constituent masses of the light and strange quarks in the evaluation of the relativistic correction for strangeness changing decays $\left(\bar{m}_{q}=\sqrt{340 \cdot 460} \mathrm{MeV}=395 \mathrm{MeV}\right)$. In this case the relativistic correction, which amounts to a reduction of $\sim 19 \%$, leads to notably better overall agreement with the empirical values, with exception of the case of $g_{A}\left(\Xi^{-} \rightarrow \Sigma^{0}\right)$. 
In column IV we show the results obtained with $g_{A}^{q}=0.87$ [9] and inclusion of the relativistic correction. These results are in very good agreement with the empirical values.

\section{The pseudoscalar exchange magnetic moment operator}

The nonrelativistic pseudoscalar octet exchange interaction between two constituent quarks has the following general expression in momentum space:

$$
V(\vec{k})=-v(\vec{k}) \vec{\sigma}^{1} \cdot \vec{k} \vec{\sigma}^{2} \cdot \vec{k} \vec{\lambda}^{1} \cdot \vec{\lambda}^{2}
$$

in the $S U(3)_{F}$ symmetric limit. Here $\vec{k}$ is the momentum transfer and $v(\vec{k})$ a scalar potential function. The exchange current operator, which satisfies the continuity equation with this interaction, yields - to lowest order in $v / c$ - the magnetic moment operator [3, 15]:

$$
\begin{gathered}
\vec{M}_{e x}=\frac{1}{2}\left\{\left(\vec{\tau}^{1} \times \vec{\tau}^{2}\right)_{3}+\lambda_{4}^{1} \lambda_{5}^{2}-\lambda_{5}^{1} \lambda_{4}^{2}\right\} \\
\left\{v(k) \vec{\sigma}^{1} \times \vec{\sigma}^{2}-\frac{1}{k} \frac{\partial v(k)}{\partial k} \vec{k} \times\left(\vec{k} \times\left(\vec{\sigma}^{1} \times \vec{\sigma}^{2}\right)\right)\right\},
\end{gathered}
$$

to which also should be added a term proportional to the center-of-mass coordinate, but which does not contribute to $S$-state quarks.

For the ground state baryons, we shall only need to consider the spatial scalar components of the interaction (3.1) and exchange magnetic moment operator (3.2), which are

$$
\begin{gathered}
V_{S}(\vec{k})=-\frac{1}{3} v(k) k^{2} \vec{\sigma}^{1} \cdot \vec{\sigma}^{2} \vec{\lambda}^{1} \cdot \vec{\lambda}^{2}, \\
\vec{M}_{S}=\frac{1}{2}\left\{\left(\vec{\tau}^{1} \times \vec{\tau}^{2}\right)_{3}+\lambda_{4}^{1} \lambda_{5}^{2}-\lambda_{5}^{1} \lambda_{4}^{2}\right\} \\
\left\{v(k)+\frac{2}{3} k \frac{\partial v(k)}{\partial k}\right\} \vec{\sigma}^{1} \times \vec{\sigma}^{2} .
\end{gathered}
$$

Fourier transformation to configuration space yields

$$
V_{S}(\vec{r})=\frac{1}{3} f(r) \vec{\sigma}^{1} \cdot \vec{\sigma}^{2} \vec{\lambda}^{1} \cdot \vec{\lambda}^{2},
$$




$$
\vec{M}_{S}=-\frac{1}{2} g(r)\left\{\left(\vec{\tau}^{1} \times \vec{\tau}^{2}\right)_{3}+\lambda_{4}^{1} \lambda_{5}^{2}-\lambda_{5}^{1} \lambda_{4}^{2}\right\} \vec{\sigma}^{1} \times \vec{\sigma}^{2},
$$

where we have used the notation

$$
\begin{gathered}
f(r)=\nabla^{2} \tilde{v}(r), \\
g(r)=\tilde{v}(r)+\frac{2}{3} \vec{r} \cdot \vec{\nabla} \tilde{v}(r),
\end{gathered}
$$

and where $\tilde{v}(r)$ is the Fourier transform of the potential function $v(k)$ :

$$
\tilde{v}(r)=\frac{1}{2 \pi^{2} r} \int_{0}^{\infty} d k k \sin (k r) v(k) .
$$

By way of illustration we note that for a bare pseudoscalar meson exchange interaction the potential function $v(k)$ is

$$
v(k)=\frac{g^{2}}{4 m_{1} m_{2}} \frac{1}{\mu^{2}+k^{2}}
$$

where $m_{1}$ and $m_{2}$ are the constituent masses of the two interacting quarks, $\mu$ is the meson mass and $g$ is the meson-quark coupling constant.

Insertion of this expression in (3.5) and (3.6) yields the following expressions for the functions $f(r)$ and $g(r)$ :

$$
\begin{aligned}
& f(r)=\frac{g^{2}}{4 \pi} \frac{1}{4 m_{1} m_{2}}\left\{\mu^{2} \frac{e^{-\mu r}}{r}-4 \pi \delta(\vec{r})\right\} \\
& g(r)=-\frac{g^{2}}{4 \pi} \frac{\mu}{12 m_{1} m_{2}}(2 \mu r-1) \frac{e^{-\mu r}}{\mu r}
\end{aligned}
$$

These bare pseudoscalar exchange operators do not take into account the spatially extended structure of the constituent quarks and should be modified accordingly at short distances prior to comparison with data.

The volume of the function $f(r)$ in (3.8a) vanishes. This is a consequence of the fact that the coupling of Goldstone bosons to quarks vanishes with momentum, as implied by the expression (3.3a), by which $V_{S}(\vec{k}=0)=0$. As $V_{S}(\vec{k}=0)$ is proportional to the volume integral of $f(r)$, the latter also 
vanishes.

In practice the potential function $f(r)$ will be determined by fits to the baryon spectra. One then needs an algorithm for determining the function $g(r)$, given $f(r)$. This is obtained by solving (3.5a) for $\tilde{v}(r)$ and then inserting the result in $(3.5 \mathrm{~b})$. The sought for expression for $g(r)$ is then

$$
g(r)=-\frac{1}{3}\left\{2 \int_{r}^{\infty} d r^{\prime} r^{\prime} f\left(r^{\prime}\right)-\frac{1}{r} \int_{r}^{\infty} d r^{\prime} \int_{r^{\prime}}^{\infty} d r^{\prime \prime} r^{\prime \prime} f\left(r^{\prime \prime}\right)\right\}
$$

It is readily seen that insertion of the example function (3.8a) in this expression yields the correct answer (3.8b) for $g(r)$. If the volume integral of the phenomenologically determined model function $f(r)$ does not vanish an inconsistency in the expressions for the interaction and exchange current operators above appears, however. This implies that part of the effective spin-flavor interaction (3.4a) would not have its origin in pseudoscalar exchange mechanisms, but in exchange mechanisms of shorter range as e.g. axial vector exchange, which does not contribute to the exchange magnetic moment in lowest order. The corresponding exchange magnetic moment operator (3.4b) constructed with $g(r)$ determined by (3.9) in this case has to be renormalized down by the fraction of the phenomenological interaction that is due to axial vector exchange, which does not give rise to any exchange moment to lowest order [15].

In ref. [4] it has been shown that a very satisfactory prediction for the spectra of the nucleon and the $\Delta$-resonance is obtained if the confining potential between the quarks is taken to be linear $\left(0.474 \mathrm{fm}^{-2} r\right)$ and the function $f(r)$ in the pseudoscalar exchange interaction (3.4a) is taken to have the form

$$
f(r)=\frac{g^{2}}{4 \pi} \frac{1}{4 m_{1} m_{2}}\left\{\mu^{2} \frac{e^{-\mu r}}{r} H(r)-\frac{4}{\sqrt{\pi}} \alpha^{3} e^{-\alpha^{2}\left(r-r_{0}\right)^{2}}\right\} .
$$

Here $H(r)$ is a function of the form

$$
H(r)=\left\{1-\frac{1}{1+e^{\beta\left(r-r_{0}\right)}}\right\}^{5},
$$

which cuts off the Yukawa function at $r_{0}=0.43 \mathrm{fm}$. The value of the parameter $\beta$ is $20 \mathrm{fm}^{-1}$. In (3.10) the $\delta$-function has been smeared over a range 
$\alpha^{-1}$, where $\alpha=2.91 \mathrm{fm}^{-1}$, which corresponds to the radius of the constituent quarks. The $\pi$-quark coupling constant $g$ is $g^{2} / 4 \pi=0.67$, which corresponds to the value $g_{\pi N N}^{2} / 4 \pi=14.2$ [3]. The volume integral of the function $f(r)$ does not vanish. The ratio of the volume integral of the negative inner and the positive outer part of $f(r)(f(1.26 \mathrm{fm})=0)$ is 10.6 . This suggests that only the fraction $X=1 / 10.6 \simeq 0.094$ of the short range part of the interaction should be interpreted as arising from Goldstone boson exchange (the outer part has the Yukawa tail, which corresponds to Goldstone boson exchange). We shall therefore multiply the calculated exchange magnetic moments with this factor $X$. The remaining fraction $1-X=0.906$ of the negative part of the phenomenological interaction (3.10) we interpret as being due to axial vector exchange mechanisms for which there is no requirement on the volume integral, and for which exchange current corrections appear only as relativistic corrections. These will be treated in the following subsection. We shall show that when $g(r)$ is calculated from the expression (3.9), with the model function (3.10) renormalized as mentioned above (using for simplicity the unit step function $\theta\left(r-r_{0}\right)$ for $\left.H(r)\right)$, the corresponding exchange current correction to the magnetic moments of the baryons go in the right direction for compensating the relativistic corrections, except in the case of the decuplet baryons that have symmetric flavor wave functions and those octet baryons for which all the 3 quarks have equal charge as the $\Sigma^{-}$and $\Xi^{-}$.

In Table 3 we list the matrix elements of the flavor-spin operators $(F, S)_{1}$ $=\left(\vec{\tau}^{1} \times \vec{\tau}^{2}\right)_{3}\left(\vec{\sigma}^{1} \times \vec{\sigma}^{2}\right)$ and $(F, S)_{2}=\left(\lambda_{4}^{1} \lambda_{5}^{2}-\lambda_{5}^{1} \lambda_{4}^{2}\right)\left(\vec{\sigma}^{1} \times \vec{\sigma}^{2}\right)_{3}$ in the exchange magnetic moment operator (3.2) for the octet baryons and the $\Delta \rightarrow N$ transition magnetic moment. Because of the antisymmetry of the flavor parts of these operators they have no matrix element for the baryon decuplet.

The contributions to the baryon magnetic moments of the exchange current operator (3.4b) can then be expressed (in units of nuclear magnetons) as

$$
\mu^{e x}=-\frac{1}{2 m_{N}} m_{N}<g(r)_{g . s .}(F, S)>=-\frac{1}{2 m_{N}} m_{N}<g(r)>_{g . s .}<(F, S)>,
$$

where $m_{N}$ is the nucleon mass, $\langle g(r)\rangle_{g . s}$ is the spatial matrix element of the function $g(r)$ for the ground state and the matrix elements of the flavor-spin operator $(F, S)$ are those given in Table 3. The first expression 
for $\mu^{e x}$ in eq. (3.12) should be used with the 3 quark wave function of ref. [4], while the latter expression in eq. (3.12) can be used with the oscillator model. Since $g(r)$ is dependent on the quark masses $m_{1}$ and $m_{2}$ (eqs. (3.9) and (3.10)), the appropriate masses should be taken into account when calculating $<g(r)>_{\text {g.s. }}$. The masses $m_{1}=m_{2}=340 \mathrm{MeV}$ are used together with the flavor-spin matrix elements $\left\langle(F, S)_{1}>\right.$, while the masses $m_{1}=340$ $\mathrm{MeV}$ and $m_{2}=460 \mathrm{MeV}$ should be used with $<(F, S)_{2}>$.

For the bare pion exchange potential (3.7) the matrix element $<g(r)>_{g . s .}$ is very small and positive, which leads to a small negative value for this exchange current contribution to the proton magnetic moment. The short range modification of the meson exchange potential leads to a change of sign of this contribution [6]. The pseudoscalar exchange potential with the short range modification (3.10) leads to values for the matrix element $m_{N}<g(r)>_{g . s}$. in (3.12) which are strongly dependent on the parameter $r_{0}$ in the short range modification (3.10) as well as on the size of the baryon wave function. With $\omega_{\text {eff }}=540 \mathrm{MeV}$ in the oscillator model, and using the same parameter values in the pseudoscalar potential (3.10) as in ref. [4], we obtain $m_{N}<g(r)>_{g . s .}=0.056$ (after multiplication by the renormalization factor $X=0.094)$. This implies an exchange current correction to the proton magnetic moment of 0.22 n.m., With the exact wave function of ref. [4] the value of the exchange current correction is slightly larger $(0.23$ n.m.). As this exchange current contribution was calculated without account of relativistic corrections, it is expected to represent an overestimate.

The main relativistic correction to the pseudoscalar exchange magnetic moment operator $(3.4 \mathrm{~b})$ can be inferred from the expansion to order $1 / m_{q}^{4}$ of the general pseudoscalar exchange magnetic moment operator in ref. [15]. This correction can to a good approximation be taken into account as an overall correction factor $\left(1-4 \vec{v}^{2} / 3\right)$. As the origin of this factor is the spinor normalization factor and the energy denominator in the small components of the quark wave functions, it is most properly taken into account in unexpanded form as an overall factor $\left(1+4 \vec{v}^{2} / 3\right)$ in the denominator of the expression $(3.4 \mathrm{~b})$ for the exchange current operator. This correction factor reduces the estimate of the pseudoscalar exchange contribution to the magnetic moment of the proton in the oscillator model by a factor 0.46 from 0.22 n.m. to the smaller value 0.10 n.m. 
The exchange current corrections to the magnetic moments of the octet baryons calculated and renormalized in this way are listed in column "EXCI" in Table 4.

\section{The flavor symmetric pseudoscalar exchange current}

The flavor antisymmetric exchange magnetic moment operator (3.2) considered above is associated with the static nonrelativistic pseudoscalar exchange interaction (3.1). When the exchange current operator that is associated with the relativistic form of the pseudoscalar exchange interaction is expanded in powers of $v / c$, flavor symmetric terms appear in the order $m_{q}^{-4}$. The corresponding magnetic moment operator has been given for the case of the two-nucleon system in ref. [15]. Applying the results of ref. [15] to the case of two quarks that interact by exchange of the $S U(3)_{F}$ octet of pseudoscalar mesons yields an exchange magnetic moment operator that can be decomposed into flavor symmetric and flavor antisymmetric parts as

$$
\vec{M}_{e x}=\vec{M}_{e x}^{F, S}+\vec{M}_{e x}^{F, A} .
$$

The flavor symmetric exchange magnetic moment operator can be written as

$$
\begin{gathered}
\vec{M}_{e x}^{F, S}=\frac{1}{8 m_{1} m_{2}} v(k)\left(\vec{\sigma}^{1}+\vec{\sigma}^{2}\right) \cdot \vec{k} \vec{k} \\
{\left[\frac{2}{3} \vec{\tau}^{1} \cdot \vec{\tau}^{2}+\left(\tau^{1}+\tau^{2}\right)_{3}+\frac{2}{3}\left(\lambda_{4}^{1} \lambda_{4}^{2}+\lambda_{5}^{1} \lambda_{5}^{2}\right)\right.} \\
-\frac{4}{3}\left(\lambda_{6}^{1} \lambda_{6}^{2}+\lambda_{7}^{1} \lambda_{7}^{2}\right)-\frac{2}{3} \lambda_{8}^{1} \lambda_{8}^{2} \\
\left.+\frac{2}{3 \sqrt{3}}\left(\lambda_{8}^{1}+\lambda_{8}^{2}\right)+\frac{1}{\sqrt{3}}\left(\lambda_{3}^{1} \lambda_{8}^{2}+\lambda_{8}^{1} \lambda_{3}^{2}\right)\right],
\end{gathered}
$$

where $v(k)$ is the pseudoscalar interaction potential defined in (3.1). The flavor antisymmetric term is correspondingly

$$
\vec{M}_{e x}^{F, A}=\frac{1}{8 m_{1} m_{2}} v(k)\left(\vec{\sigma}^{1}-\vec{\sigma}^{2}\right) \cdot \vec{k} \vec{k}
$$




$$
\begin{gathered}
{\left[\left(\vec{\tau}^{1}-\vec{\tau}^{2}\right)_{3}+\frac{2}{3 \sqrt{3}}\left(\lambda_{8}^{1}-\lambda_{8}^{2}\right)\right.} \\
\left.+\frac{1}{\sqrt{3}}\left(\lambda_{8}^{1} \lambda_{3}^{2}-\lambda_{3}^{1} \lambda_{8}^{2}\right)\right]
\end{gathered}
$$

in addition to a term with the same flavor operator as the main flavor antisymmetric exchange magnetic moment operator (3.2), and which may be viewed as a relativistic correction to it. The importance of the flavor symmetric exchange magnetic moment operator (4.2) derives from the fact that it gives a nonvanishing contribution to the magnetic moments of the decuplet baryons as well as the $\Sigma^{-}$and $\Xi^{-}$octet hyperons, to which the flavor antisymmetric terms cannot contribute.

In the case of the ground state baryons, which have all constituent quarks in the lowest $S$-state, only the spatial scalar components of the spin operators in (4.2) and (4.3) contribute. By retaining only that and taking the Fourier transform yields the exchange magnetic moment operators

$$
\begin{gathered}
\vec{M}_{e x}^{F, S}=-\frac{1}{24 m_{1} m_{2}} f(r)\left(\vec{\sigma}^{1}+\vec{\sigma}^{2}\right)_{3}\left[\frac{2}{3} \vec{\tau}^{1} \cdot \vec{\tau}^{2}+\left(\vec{\tau}^{1}+\vec{\tau}^{2}\right)_{3}\right. \\
+\frac{2}{3}\left(\lambda_{4}^{1} \lambda_{4}^{2}+\lambda_{5}^{1} \lambda_{5}^{2}\right) \\
-\frac{4}{3}\left(\lambda_{6}^{1} \lambda_{6}^{2}+\lambda_{7}^{1} \lambda_{7}^{2}\right)-\frac{2}{3} \lambda_{8}^{1} \lambda_{8}^{2} \\
\left.+\frac{2}{3 \sqrt{3}}\left(\lambda_{8}^{1}+\lambda_{8}^{2}\right)+\frac{1}{\sqrt{3}}\left(\lambda_{3}^{1} \lambda_{8}^{2}+\lambda_{8}^{1} \lambda_{3}^{2}\right)\right], \\
\vec{M}_{e x}^{F, A}=-\frac{1}{24 m_{1} m_{2}} f(r)\left(\vec{\sigma}^{1}-\vec{\sigma}^{2}\right)_{3}\left[\left(\vec{\tau}^{1}-\vec{\tau}^{2}\right)_{3}\right. \\
\left.+\frac{2}{3 \sqrt{3}}\left(\lambda_{8}^{1}-\lambda_{8}^{2}\right)+\frac{1}{\sqrt{3}}\left(\lambda_{8}^{1} \lambda_{3}^{2}-\lambda_{3}^{1} \lambda_{8}^{2}\right)\right] .
\end{gathered}
$$

Here the function $f(r)$ is the Laplacian of the pseudoscalar exchange interaction, as defined in eq. (3.5a). Note that the factor $\frac{1}{m_{1} m_{2}}$ in eqs. (4.4a) and $(4.4 \mathrm{~b})$ is not the same in all parts of the expressions. In the calculations we will use the masses $m_{1}=m_{2}=340 \mathrm{MeV}$ for the non-strange baryons and $m_{1}=m_{2}=460 \mathrm{MeV}$ for the $\Omega^{-}$hyperon. In all other cases we make 
a decomposition of the expressions for $\vec{M}_{e x}^{F, S}$ and $\vec{M}_{e x}^{F, A}$ so that terms with flavor operators containing $\tau$ have $m_{1}=m_{2}=340 \mathrm{MeV}$ and other terms have $m_{1}=340 \mathrm{MeV}, m_{2}=460 \mathrm{MeV}$. When using the oscillator model the calculation of $\left\langle\vec{M}_{e x}^{F, S}>\right.$ and $\left\langle\vec{M}_{e x}^{F, A}\right\rangle$ can be simplified as described below.

The fact that the radial behaviour of the exchange magnetic moment operators is directly proportional to the pseudoscalar exchange interaction implies that the corresponding magnetic moments of the ground state baryons may for the oscillator model be expressed in terms of the orbital matrix element of the pseudoscalar exchange interaction for the ground state baryons. If the whole effective interaction (3.4a) were due to pseudoscalar exchange, this matrix element would, as defined in ref. [3], be

$$
P_{00}=-\frac{1}{3}<f(r)>_{g . s .} .
$$

In first order perturbation theory the matrix element $P_{00}$ may be determined directly from the empirical $N-\Delta$ splitting, which in that approximation is simply $10 P_{00}$, so that $P_{00} \simeq 29 \mathrm{MeV}$ [3]. With the model (3.10) for the radial shape of the fine structure interaction the matrix element calculated with the oscillator model $\left(\omega_{e f f}=540 \mathrm{MeV}\right)$ is as large as $101 \mathrm{MeV}$. Note that only the fraction $X=0.094$ of this matrix element can - as explained above - be ascribed to pseudoscalar exchange. The relativistic correction to the proton exchange magnetic moment would then be 0.15 n.m. (0.17 n.m. with the exact wave function of ref. [4]). If as in section 3 above we include a factor $\left(1+4 \vec{v}^{2} / 3\right)$ in the denominators of the expressions (4.2) and (4.3) (or alternatively in the expression (4.5)) to account for relativistic corrections from the Dirac operators this estimate for the matrix element $P_{00}$ in the oscillator model drops to only $46 \mathrm{MeV}$. This value for $P_{00}$ has still to be renormalized down, however, in view of the fact that the volume integral of the phenomenological potential function (3.10) does not vanish, as would be required by pseudoscalar exchange mechanisms. For the $K$ meson exchange contribution the corresponding matrix element is calculated as in eq. (4.5), but with the average mass $\bar{m}_{q}=395 \mathrm{MeV}$. When including the factor $1 /\left(1+4 \vec{v}^{2} / 3\right)$, also calculated with $\bar{m}_{q}$, one gets $41 \mathrm{MeV}$. Denoting these two matrix elements $P_{00}^{\pi}$ and $P_{00}^{K}$ and assuming that the $\eta$ meson exchange contribution is $P_{00}^{\eta}=P_{00}^{K}$ one may express the orbital matrix elements of the 
exchange magnetic moments (4.4) as

$$
\begin{gathered}
<\vec{M}_{e x}^{F, S}>=\mu_{N} X\left(\frac{m_{N}}{4 m_{1} m_{2}}\right)\left(\vec{\sigma}^{1}+\vec{\sigma}^{2}\right)_{3} \\
\left\{\left[\frac{2}{3} \vec{\tau}^{1} \cdot \vec{\tau}^{2}+\left(\vec{\tau}^{1}+\vec{\tau}^{2}\right)_{3}\right] P_{00}^{\pi}\right. \\
+\left[\frac{2}{3}\left(\lambda_{4}^{1} \lambda_{4}^{2}+\lambda_{5}^{1} \lambda_{5}^{2}\right)-\frac{4}{3}\left(\lambda_{6}^{1} \lambda_{6}^{2}+\lambda_{7}^{1} \lambda_{7}^{2}\right)\right] P_{00}^{K} \\
\left.-\left[\frac{2}{3} \lambda_{8}^{1} \lambda_{8}^{2}-\frac{2}{3 \sqrt{3}}\left(\lambda_{8}^{1}+\lambda_{8}^{2}\right)-\frac{1}{\sqrt{3}}\left(\lambda_{3}^{1} \lambda_{8}^{2}+\lambda_{8}^{1} \lambda_{3}^{2}\right)\right] P_{00}^{\eta}\right\}, \\
<\vec{M}_{e x}^{F, A}>=\mu_{N} X\left(\frac{m_{N}}{4 m_{1} m_{2}}\right)\left(\vec{\sigma}^{1}-\vec{\sigma}^{2}\right)_{3} . \\
\left\{\left(\vec{\tau}^{1}-\vec{\tau}^{2}\right)_{3} P_{00}^{\pi}\right. \\
\left.+\frac{1}{\sqrt{3}}\left[\frac{2}{3}\left(\lambda_{8}^{1}-\lambda_{8}^{2}\right)+\left(\lambda_{8}^{1} \lambda_{3}^{2}-\lambda_{3}^{1} \lambda_{8}^{2}\right)\right] P_{00}^{\eta}\right\} .
\end{gathered}
$$

The matrix elements of the flavor-spin operators in these two expressions are listed in Table 5. The factor $X$ has the value 0.094 .

The contributions to the baryon magnetic moments of the exchange current operators (4.6) can be expressed as $\mu_{r e l}^{e x}=\mu_{N}\left(m_{N} / 4 m_{1} m_{2}\right)$ times the sum of the flavor-spin matrix elements in Table 5. As in eq (4.4) the masses $m_{1}$ and $m_{2}$ should be taken as $m_{1}=m_{2}=340 \mathrm{MeV}$ for non-strange baryons and for strange baryons when associated with the matrix element $P_{00}^{\pi}$, as $m_{1}=m_{2}=460 \mathrm{MeV}$ for the $\Omega^{-}$hyperon and otherwise as $m_{1}=340 \mathrm{MeV}$ and $m_{2}=460 \mathrm{MeV}$. The numerical values obtained in this way for the pseudoscalar exchange magnetic moments of order $m^{-4}$ are given in column EXCII in Table 4.

The results in Table 4 show that the typical magnitude of the contributions to the baryon magnetic moments of the relativistic corrections to the exchange current operators are somewhat smaller than that of the static exchange current operator (3.12) considered in the previous section. They also in every instance go in the right direction to improve the agreement between the quark model predictions and the empirical values for the magnetic moment operators. In the case of the decuplet baryons it is only the relativistic 
exchange current that gives a contribution and thus it is important for the compensation of the relativistic corrections.

\section{Exchange magnetic moment for phenomenological interac- tions}

As the volume integral of the phenomenological interaction model (3.10) [4] does not vanish, a fraction $(1-X)$ of the short range part of it has to be interpreted as arising from other than pseudoscalar exchange mechanisms.

If the phenomenological quark-quark interaction is expressed in terms of the usual 5 Fermi invariants, only the tensor $(T)$ and axial vector $(A)$ invariants give rise to spin-spin interactions of the form $(3.4 \mathrm{a})$, that have non-vanishing volume integrals in lowest order in $1 / m$ [19]. Since the form of these interactions and the corresponding exchange current operators are very similar, we restrict the consideration here to the axial vector invariant.

While the spin-spin interactions, which are associated with the $T$ and $A$ invariants, are of order $m^{0}$, the corresponding exchange magnetic moment operators are of order $m^{-2}$ - i.e. they have the form of relativistic corrections. If the spin-spin interaction has the form (3.4a), and is assumed to arise from an interaction with the operator structure of the $A$ invariant, the contribution to lowest order in $1 / m$ to the corresponding exchange magnetic moment is

$$
\begin{aligned}
\vec{M}_{e x}(A)=-\frac{(1-X)}{24 m_{1} m_{2}} f(r)\left\{( \vec { \sigma } ^ { 1 } + \vec { \sigma } ^ { 2 } ) _ { 3 } \left[\frac{2}{3} \vec{\tau}^{1} \cdot \vec{\tau}^{2}+\left(\vec{\tau}^{1}+\vec{\tau}^{2}\right)_{3}\right.\right. \\
+\frac{2}{3}\left(\lambda_{4}^{1} \lambda_{4}^{2}+\lambda_{5}^{1} \lambda_{5}^{2}\right) \\
\quad-\frac{4}{3}\left(\lambda_{6}^{1} \lambda_{6}^{2}+\lambda_{7}^{1} \lambda_{7}^{2}\right)-\frac{2}{3} \lambda_{8}^{1} \lambda_{8}^{2} \\
\left.+\frac{2}{3 \sqrt{3}}\left(\lambda_{8}^{1}+\lambda_{8}^{2}\right)+\frac{1}{\sqrt{3}}\left(\lambda_{3}^{1} \lambda_{8}^{2}+\lambda_{8}^{1} \lambda_{3}^{2}\right)\right] \\
+\left(\vec{\sigma}^{1}-\vec{\sigma}^{2}\right)_{3}\left[\left(\vec{\tau}^{1}-\vec{\tau}^{2}\right)_{3}+\frac{2}{3 \sqrt{3}}\left(\lambda_{8}^{1}-\lambda_{8}^{2}\right)\right.
\end{aligned}
$$




$$
\begin{gathered}
\left.+\frac{1}{\sqrt{3}}\left(\lambda_{8}^{1} \lambda_{3}^{2}-\lambda_{3}^{1} \lambda_{8}^{2}\right)\right] \\
\left.-2\left(\vec{\sigma}^{1} \times \vec{\sigma}^{2}\right)\left[\left(\vec{\tau}^{1} \times \vec{\tau}^{2}\right)_{3}+\lambda_{4}^{1} \lambda_{5}^{2}-\lambda_{5}^{1} \lambda_{4}^{2}\right]\right\} .
\end{gathered}
$$

From this expression a small term involving the derivative of $f(r)$ has been left out. With exception for the last term in the bracket, this operator is identical in form to the relativistic pseudoscalar exchange operators (4.4a) and (4.4b). The overall factor $1-X=0.906$ gives the fraction of the short range part of the phenomenological interaction (3.10), which is interpreted as arising from axial vector exchange mechanisms. The expression (5.1) for the magnetic moment operator associated with the axial vector invariant was obtained by generalizing the corresponding $S U(2)$ expressions given in ref. 15] to $S U(3)$ and Fourier transforming the resulting expression.

In view of the similarity in form between this expression and the expressions (4.4), its matrix elements for the ground state can be obtained by the same method as used in going from the expressions (4.4) to the corresponding matrix element expressions (4.6). In this case the indices $\pi, K$ and $\eta$ on the radial integrals $P_{00}$ (4.6) only indicate that the integrals are assumed to depend on the quark masses in the same way as the matrix elements of $\pi, K$ and $\eta$ exchange interactions. The contributions to the baryon magnetic moments given by the magnetic moment expressions (5.1) calculated in this way and using the same numerical values as in the expressions (4.4) are listed in the column EXCIII in Table 4. The required matrix elements of the spinflavor operators are given in Tables 3 and 5.

It is worth noting, that while the pseudoscalar exchange mechanisms give rise to an axial exchange current operator, which is only of order $m^{-5}$, axial exchange mechanisms give rise to exchange currents of order $m^{-3}$ [18]. While the contributions of the former represent 4th order relativistic corrections, and thus should be insignificant, the latter may give rise to non-negligible contributions to the axial coupling constants of the baryons. A quantitative calculation of these would require a completely covariant framework, and is therefore not attempted here. 


\section{Discussion}

The results in Tables 2 and 4 show that the corrections to the baryon magnetic moments that arise from exchange current operators are large, when using the phenomenological interaction model of ref. [G], which leads to a very satisfactory baryon spectrum, and that they tend to compensate the large relativistic corrections to the static quark model predictions for the magnetic moments of the baryons. The absence of pure pseudoscalar exchange currents of low order to the axial current of the baryons on the other hand implies that the corresponding if somewhat smaller relativistic corrections to the axial coupling constants of the baryons remain uncompensated. The present results, which are based on the hyperfine interaction model of ref. [4], cannot, however, be viewed as being very satisfactory in view of the fact that the resulting predictions for the baryon magnetic moments typically exceed the empirical values by about $20 \%$. This points to the need for a consistent relativistic treatment as well as more information on the shorter range vector and axial exchange contributions to the phenomenological interaction. It should be stressed that the meson exchange type description of the hyperfine interaction between the constituent quarks, which leads to very satisfactory

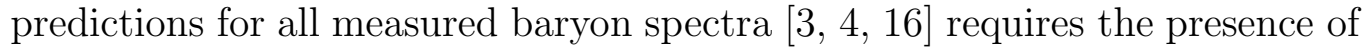
these magnetic moment operators through the continuity equation because of the flavor dependence of the interaction. On the other hand the fact that the relativistic corrections to the exchange current operator considered in section 4 are only slightly smaller than the non-relativistic static exchange current contributions considered in section 3 emphasices the need for a fully relativistic treatment.

The treatment of the pseudoscalar octet exchange current contributions to the magnetic moments of the baryons differs from previous work [6, [7] in that we consider the full octet exchange current operator, and in the treatment of the short range part of the pseudoscalar exchange interaction, and finally in that we also consider the lowest order relativistic corrections to the exchange current operator. In ref. [6] the exchange currents associated with the one gluon exchange interaction between the constituent quarks was also considered, but this is likely to be less significant than what was suggested in ref. [6] in view of the insignificance of the one gluon exchange interaction that is indicated by the baryon spectrum [3]. 
In addition to the pseudoscalar octet exchange current operators that have been considered here it would be natural to also consider the exchange currents that are mediated by the scalar (" $\sigma$-meson") partner of the pseudoscalar octet of Goldstone bosons. The corresponding operator can be obtained from the scalar meson exchange current operator given in refs. [15, 18] by appropriate replacements of the nucleon mass and coupling constants by the quark mass and coupling constants. The fact that the impulse approximation results for the axial coupling constants of the baryons agree fairly well with the empirical values, once the relativistic corrections are taken into account, suggests that this scalar exchange current contribution should be small. A natural way to understand this result is to assume that there is a strong cancellation between the scalar meson exchange current contributions to both the magnetic moments and the axial coupling constants and the corresponding exchange current contributions that are expected to be associated with the linear confining interaction, which will have the opposite sign [6].

Finally it should be noted that the fraction of the phenomenological interaction (3.4a) could also due to vector instead of pseudoscalar and axial exchange mechanisms. The spin-spin component of the vector exchange interaction has to have zero volume integral as does the corresponding pseudoscalar model (3.10). In the case of vector exchange mechanisms, the relation $(3.5 \mathrm{~b})$ between the orbital part of the magnetization density should be replaced by

$$
g(r)=2 \tilde{v}(r)+\vec{r} \cdot \vec{\nabla} \tilde{v}(r) .
$$

If this relation were used in place of the relation (3.5b) the exchange current contributions in the column EXCI of Table 4 would be somewhat reduced.

The separation of the exchange magnetic moment operator here into a term that was associated with the pseudoscalar octet exchange mechanism, and another purely phenomenological term was motivated by the fact that the phenomenological interaction model developed in ref. 泪 has a large volume integral, whereas it should vanish for a pseudoscalar exchange interaction. Given only a spin-flavor interaction of the form (3.4a), (3.10), does not however permit a unique determination of the fraction of it that may be 
asscribed to pseudoscalar meson exchange, nor does it indicate what the dynamical origin of the short range terms that lead to the nonvanishing volume integral is. Hence there remains a substantial uncertainty as to the proper form of the exchange current operator, as the continuity equation determines only its longitudinal part. In view of this uncertainty, we view the predicted exchange current contributions in Table 4 as no more than suggestive, and expect them to be very strongly dependent on the particular form of the phenomenological interaction employed here. As a concrete example of this, the volume integral of the interaction (3.10) is very sensitive to the parameter $r_{0}$ in (3.10) and be reduced by $50 \%$ by a small reduction of the value for $r_{0}$. The very small fraction of the phenomenological interation used here, which can be ascribed to pseudoscalar exchange mechanisms is due to the fact that it changes sign at the probably unrealistically large radius $1.26 \mathrm{fm}$, whereas for copmparison the corresponding isospin dependent spin-spin interaction component in a recently developed realistic phenomenological nucleon-nucleon interaction model [20] changes sign already at $0.6 \mathrm{fm}$.

\section{Acknowledgement}

We thank Dr. L. Ya. Glozman for supplying the wave function for the ground state baryons for the interaction model in ref. [4], and for critical correspondence. DOR thanks Professor M. Rosina for an instructive discussion and Professor V. Vento for valuable correspondence. 


\section{References}

[1] F. Close, An Introduction to Quarks and Partons, Academic Press, London (1979)

[2] C. Hayne and N. Isgur, Phys. Rev. D25 (1982) 1944

[3] L.Ya. Glozman and D.O. Riska, Phys. Reports 268 (1996) 263

[4] L.Ya. Glozman, Z. Papp and W. Plessas, Phys. Lett. B381 (1966) 311

[5] T.P. Cheng and L.-F. Li, Phys. Rev. Lett. 74 (1995) 2872

[6] A. Buchmann, E. Hernández and K. Yazaki, Nucl. Phys. A569 (1994) 661

[7] S. Weinberg, Phys. Rev. Lett. 65 (1990) 1181

[8] S. Weinberg, Phys. Rev. Lett. 67 (1991) 3473

[9] D.A. Dicus et al., Phys. Lett. B284 (1992) 384

[10] Particle Data Group, Phys. Rev. D50 (1994) 1173

[11] A. Bosshard et al., Phys. Rev. D44 (1991) 1962

[12] N.B. Wallace et al., Phys. Rev. Lett. 74 (1995) 3732

[13] L. Brekke, Ann. Phys. 240 (1995) 400

[14] I.M. Gaillard and G. Sauvage, Ann. Rev. Nucl. Part. Sci. 34 (1984) 351

[15] K. Tsushima, D.O. Riska and P.G. Blunden, Nucl. Phys. A559 (1993) 543

[16] L.Ya. Glozman and D.O. Riska, Nucl.Phys. A603 (1966) 326

[17] D. Robson, Nucl. Phys. A560 (1993) 389

[18] K. Tsushima and D.O. Riska, Nucl. Phys. A549 (1992) 313

[19] P.G. Blunden and D.O. Riska, Nucl. Phys. A536 (1992) 697

[20] R. B. Wiringa, V. G. J.Stoks and R. Schiavilla, Phys. Rev. C 51 (1995) 38 


\section{Table 1}

Magnetic moments of the ground state baryon octet and the $\Delta^{++}$and $\Omega^{-}$(in nuclear magnetons). Column IA contains the quark model impulse approximation expressions with the relativistic corrections. Columns I and II contain the impulse approximation values with and without the relativistic correction. The empirical values are from ref. [10-12].

\begin{tabular}{|c|c|c|c|c|}
\hline & IA & exp & I & II \\
\hline$p$ & $\frac{m_{N}}{m_{u}^{*}}$ & +2.79 & +2.76 & 1.80 \\
\hline$n$ & $-\frac{2}{3} \frac{m_{N}}{m_{u}^{*}}$ & -1.91 & -1.84 & -1.20 \\
\hline$\Lambda$ & $-\frac{1}{3} \frac{m_{N}}{m_{s}^{*}}$ & -0.61 & -0.67 & -0.48 \\
\hline$\Sigma^{+}$ & $\frac{8}{9} \frac{m_{N}}{m_{u}^{*}}+\frac{1}{9} \frac{m_{N}}{m_{s}^{*}}$ & +2.46 & +2.68 & 1.76 \\
\hline$\Sigma^{0}$ & $\frac{2}{9} \frac{m_{N}}{m_{u}^{*}}+\frac{1}{9} \frac{m_{N}}{m_{s}^{*}}$ & $?$ & +0.84 & 0.56 \\
\hline$\Sigma^{0} \rightarrow \Lambda$ & $-\frac{1}{\sqrt{3}} \frac{m_{N}}{m_{u}^{*}}$ & $|1.61|$ & -1.59 & -1.04 \\
\hline$\Sigma^{-}$ & $-\frac{4}{9} \frac{m_{N}}{m_{u}^{*}}+\frac{1}{9} \frac{m_{N}}{m_{s}^{*}}$ & -1.16 & -1.00 & -0.64 \\
\hline$\Xi^{0}$ & $-\frac{2}{9} \frac{m_{N}}{m_{u}^{*}}-\frac{4}{9} \frac{m_{N}}{m_{s}^{*}}$ & -1.25 & -1.51 & -1.04 \\
\hline$\Xi^{-}$ & $\frac{1}{9} \frac{m_{N}}{m_{u}^{*}}-\frac{4}{9} \frac{m_{N}}{m_{s}^{*}}$ & -0.65 & -0.59 & -0.44 \\
\hline$\Delta^{++}$ & $2 \frac{m_{N}}{m_{u}^{*}}$ & 4.52 & 5.52 & 3.60 \\
\hline$\Delta^{+} \rightarrow p$ & $\frac{2 \sqrt{2}}{3} \frac{m_{N}}{m_{u}^{*}}$ & 3.1 & 2.6 & 1.70 \\
\hline$\Omega^{-}$ & $-\frac{m_{N}}{m_{s}^{*}}$ & -2.019 & -2.01 & -1.44 \\
\hline
\end{tabular}




\section{Table 2}

The axial coupling constants of the baryon octet. Column I gives the expressions in terms of the $\mathrm{F}$ and $\mathrm{D}$ coefficients and column II the static quark model prediction (with $g_{A}^{q}=1$ ). Column III gives the predicted values with inclusion of the relativistic correction with $g_{A}^{q}=1$ and column IV the predictions with $g_{A}^{q}=0.87$. The empirical values are taken from refs. [10, 14].

\begin{tabular}{|l|l|r|r|r|r|}
\hline & I & exp & II & III & IV \\
\hline$n \rightarrow p$ & $F+D$ & 1.26 & 1.67 & 1.35 & 1.17 \\
$\Sigma^{ \pm} \rightarrow \Lambda$ & $\sqrt{\frac{2}{3}} D$ & 0.62 & 0.81 & 0.66 & 0.57 \\
$\Sigma^{-} \rightarrow \Sigma^{0}$ & $\sqrt{2} F$ & 0.67 & 0.94 & 0.76 & 0.66 \\
$\Lambda \rightarrow p$ & $-\sqrt{\frac{3}{2}}\left(F+\frac{D}{3}\right)$ & 0.88 & 1.22 & 1.01 & 0.88 \\
$\Sigma^{-} \rightarrow n$ & $-(F-D)$ & 0.34 & 0.33 & 0.28 & 0.24 \\
$\Xi^{-} \rightarrow \Lambda$ & $\sqrt{\frac{3}{2}}\left(F-\frac{D}{3}\right)$ & 0.31 & 0.40 & 0.34 & 0.30 \\
$\Xi^{-} \rightarrow \Sigma^{0}$ & $\frac{1}{\sqrt{2}}(F+D)$ & 1.36 & 1.18 & 0.97 & 0.84 \\
$\Xi^{0} \rightarrow \Sigma^{+}$ & $F+D$ & $?$ & 1.67 & 1.38 & 1.20 \\
$\Xi^{-} \rightarrow \Xi^{0}$ & $F-D$ & -0.28 & -0.33 & -0.27 & -0.23 \\
\hline
\end{tabular}




\section{Table 3}

The matrix elements of the flavor-spin operators $(F, S)_{1}=\left(\tau^{1} \times \tau^{2}\right)_{3}\left(\vec{\sigma}^{1} \times\right.$ $\left.\vec{\sigma}^{2}\right)_{3}$ and $(F, S)_{2}=\left(\lambda_{4}^{1} \lambda_{5}^{2}-\lambda_{5}^{1} \lambda_{4}^{2}\right)\left(\vec{\sigma}^{1} \times \vec{\sigma}^{2}\right)_{3}$ for the ground state baryons.

\begin{tabular}{|l|c|c|}
\hline & $\left\langle(F, S)_{1}\right\rangle$ & $\left\langle(F, S)_{2}\right\rangle$ \\
\hline$p$ & -4 & 0 \\
$\Lambda$ & 4 & 0 \\
$\Sigma^{+}$ & 0 & 2 \\
$\Sigma^{0}$ & 0 & -4 \\
$\Sigma^{0} \rightarrow \Lambda$ & $\frac{4}{\sqrt{3}}$ & $\frac{2}{\sqrt{3}}$ \\
$\Sigma^{-}$ & 0 & 0 \\
$\Xi^{0}$ & 0 & 4 \\
$\Xi^{-}$ & 0 & 0 \\
\hline$\Delta^{+} \rightarrow p$ & $-4 \sqrt{2}$ & 0 \\
\hline
\end{tabular}




\section{Table 4}

The magnetic moments of the ground state baryon octet and the $\Delta^{++}$ and the $\Omega^{-}$(in nuclear magnetons). In column IA+REL the impulse approximation values that include the relativistic corrections are given. In column EXCI the contribution from the nonrelativistic pseudoscalar exchange current operator $(3.3 \mathrm{~b})$ are given. Column EXCII contains the contribution from the relativistic correction to the pseudoscalar exchange current operator (4.6). The sums of the exchange current and impulse approximation results are listed in column Total. 


\begin{tabular}{|l|r|r|r|r|r|r|}
\hline & IA+REL & EXCI & EXCII & EXCIII & Total & exp \\
\hline$p$ & 1.80 & 0.10 & 0.07 & 1.35 & 3.32 & 2.79 \\
$n$ & -1.20 & -0.10 & -0.04 & -1.06 & -2.40 & -1.91 \\
$\Lambda$ & -0.48 & -0.05 & -0.01 & -0.35 & -0.89 & -0.61 \\
$\Sigma^{+}$ & 1.76 & 0.09 & 0.06 & 0.99 & 2.90 & 2.46 \\
$\Sigma^{0}$ & 0.56 & 0.05 & 0.02 & 0.37 & 1.00 & $?$ \\
$\Sigma^{0} \rightarrow \Lambda$ & -1.04 & -0.08 & -0.03 & -0.80 & -1.95 & $|1.61|$ \\
$\Sigma^{-}$ & -0.64 & 0 & -0.03 & -0.25 & -0.92 & -1.16 \\
$\Xi^{0}$ & -1.04 & -0.09 & -0.03 & -0.69 & -1.84 & -1.25 \\
$\Xi^{-}$ & -0.44 & 0 & -0.02 & -0.20 & -0.66 & -0.65 \\
\hline$\Delta^{++}$ & 3.60 & 0 & 0.18 & 1.76 & 5.54 & 4.52 \\
$\Delta^{+} \rightarrow p$ & 1.70 & 0.14 & 0.06 & 1.50 & 3.40 & 3.1 \\
$\Omega^{-}$ & -1.44 & 0 & -0.05 & -0.44 & -1.93 & -2.019 \\
\hline
\end{tabular}




\section{Table 5}

The matrix elements of the combinations of symmetric and antisymmetric flavor and spin operators defined in eqs. (4.6) for the ground state baryons. The spin and flavor operators are defined as follows: $S_{1}=\left(\vec{\sigma}^{1}+\vec{\sigma}^{2}\right)_{3}, S_{2}=$ $\left(\vec{\sigma}^{1}-\vec{\sigma}^{2}\right)_{3}, F_{1}^{S}=\frac{2}{3} \vec{\tau}^{1} \cdot \vec{\tau}^{2}+\left(\vec{\tau}^{1}+\vec{\tau}^{2}\right)_{3}, F_{2}^{S}=\frac{2}{3}\left(\lambda_{4}^{1} \lambda_{4}^{2}+\lambda_{5}^{1} \lambda_{5}^{2}\right)-\frac{4}{3}\left(\lambda_{6}^{1} \lambda_{6}^{2}+\lambda_{7}^{1} \lambda_{7}^{2}\right)$, $F_{3}^{S}=-\left[\frac{2}{3} \lambda_{8}^{1} \lambda_{8}^{2}-\frac{2}{3 \sqrt{3}}\left(\lambda_{8}^{1}+\lambda_{8}^{2}\right)-\frac{1}{\sqrt{3}}\left(\lambda_{3}^{1} \lambda_{8}^{2}+\lambda_{8}^{1} \lambda_{3}^{2}\right)\right], F_{1}^{A}=\left(\vec{\tau}^{1}-\vec{\tau}^{2}\right)_{3}$ and $F_{2}^{A}=\frac{1}{\sqrt{3}}\left[\frac{2}{3}\left(\lambda_{8}^{1}-\lambda_{8}^{2}\right)+\left(\lambda_{8}^{1} \lambda_{3}^{2}-\lambda_{3}^{1} \lambda_{8}^{2}\right)\right]$.

\begin{tabular}{|c|c|c|c|c|c|}
\hline & $S_{1} F_{1}^{S} P_{00}^{\pi}$ & $S_{1} F_{2}^{S} P_{00}^{K}$ & $S_{1} F_{3}^{S} P_{00}^{\eta}$ & $S_{2} F_{1}^{A} P_{00}^{\pi}$ & $S_{2} F_{2}^{A} P_{00}^{\eta}$ \\
\hline$p$ & $4 P_{00}^{\pi}$ & 0 & $\frac{4}{3} P_{00}^{\eta}$ & $4 P_{00}^{\pi}$ & $-\frac{4}{3} P_{00}^{\eta}$ \\
\hline$n$ & $-\frac{4}{3} P_{00}^{\pi}$ & 0 & $-\frac{4}{9} P_{00}^{\eta}$ & $-4 P_{00}^{\pi}$ & $\frac{4}{3} P_{00}^{\eta}$ \\
\hline$\Lambda$ & 0 & $-\frac{4}{3} P_{00}^{K}$ & $\frac{4}{9} P_{00}^{\eta}$ & 0 & $-\frac{4}{3} P_{00}^{\eta}$ \\
\hline$\Sigma^{+}$ & $\frac{32}{9} P_{00}^{\pi}$ & $\frac{8}{9} P_{00}^{K}$ & $\frac{8}{9} P_{00}^{\eta}$ & 0 & $\frac{8}{3} P_{00}^{\eta}$ \\
\hline$\Sigma^{0}$ & $\frac{8}{9} P_{00}^{\pi}$ & $-\frac{4}{9} P_{00}^{K}$ & $\frac{4}{9} P_{00}^{\eta}$ & 0 & $\frac{4}{3} P_{00}^{\eta}$ \\
\hline$\Sigma^{0} \rightarrow \Lambda$ & 0 & $-\frac{4}{\sqrt{3}} P_{00}^{K}$ & $\frac{4}{3 \sqrt{3}} P_{00}^{\eta}$ & $-\frac{4}{\sqrt{3}} P_{00}^{\pi}$ & 0 \\
\hline$\Sigma^{-}$ & $-\frac{16}{9} P_{00}^{\pi}$ & $-\frac{16}{9} P_{00}^{K}$ & 0 & 0 & 0 \\
\hline$\Xi^{0}$ & 0 & $\frac{8}{9} P_{00}^{K}$ & $-\frac{8}{3} P_{00}^{\eta}$ & 0 & $-\frac{8}{3} P_{00}^{\eta}$ \\
\hline$\Xi^{-}$ & 0 & $-\frac{16}{9} P_{00}^{K}$ & $-\frac{16}{9} P_{00}^{\eta}$ & 0 & 0 \\
\hline$\Delta^{++}$ & $16 P_{00}^{\pi}$ & 0 & $\frac{16}{3} P_{00}^{\eta}$ & 0 & 0 \\
\hline$\Delta^{+} \rightarrow p$ & $\frac{8}{3 \sqrt{2}} P_{00}^{\pi}$ & 0 & $\frac{8}{9 \sqrt{2}} P_{00}^{\eta}$ & $\frac{8}{\sqrt{2}} P_{00}^{\pi}$ & $-\frac{8}{3 \sqrt{2}} P_{00}^{\eta}$ \\
\hline$\Omega^{-}$ & 0 & 0 & $-\frac{32}{3} P_{00}^{\eta}$ & 0 & 0 \\
\hline
\end{tabular}

